\title{
REPERCUSSÕES FISIOPATOLÓGICAS E CLÍNICAS DA DOENÇA DE HASHIMOTO
}

\section{ARTIGO DE REVISÃO}

FERNANDES, Thaís Da Silva ${ }^{1}$, BARBOSA, Amanda Graziele Silva² ${ }^{2}$ SANTOS, Helen Amanda Pinto dos ${ }^{3}$, SOUSA, Maria Clara Oliveira Campos ${ }^{4}$, BRASIL, Maria Madalena Costa $^{5}$, MESQUITA, Marina Feitosa De ${ }^{6}$, BRITO, Quezia Valerio7, CASSA, Victória Albani $^{8}$, COSTA, Vinícius Sampaio ${ }^{9}$, OLIVEIRA, Igor de Sousa ${ }^{10}$, RIBEIRO, Yana Sarah Fernandes Souza ${ }^{11}$, VASCONCELOS, Gilberto Loiola de ${ }^{12}$

${ }^{1}$ Discente do curso de Medicina do Centro Universitário INTA - UNINTA.

2 Discente do curso de Medicina do Centro Universitário UNIFACID.

${ }^{3}$ Discente do curso de Saúde Coletiva da Universidade Federal do Oeste do Pará.

${ }^{4}$ Discente do curso de Medicina do Centro Universitário UNIFACID.

${ }^{5}$ Discente do curso de Medicina do Centro Universitário UNIFACID.

${ }^{6}$ Discente do curso de Saúde Coletiva do Centro Universitário Max Planck - UniMAX.

${ }^{7}$ Discente do curso de Medicina da Universidade Nilton Lins.

${ }^{8}$ Discente do curso de Medicina do Centro Universitário do Espírito Santo - UNESC.

${ }^{9}$ Discente do curso de Medicina da Faculdade de Medicina de Marília.

10 Discente do curso de Medicina da Universidade Federal de Campina Grande, Centro de Formação dos Professores, Cajazeiras - Paraíba.

${ }^{11}$ Discente do curso de Medicina do Centro Universitário INTA - UNINTA.

12 Orientador. Médico residente em Clínica Médica, formado pela Universidade Federal do Ceará.

$\mathrm{RC}: 88463$

Disponível em: https://www.nucleodoconhecimento.com.br/saude/doenca-de-hashimoto 
FERNANDES, Thaís Da Silva. Repercussões fisiopatológicas e clínicas da doença de Hashimoto. Revista Científica Multidisciplinar Núcleo do Conhecimento. Ano 06, Ed. 06, Vol. 07, pp. 155-168. Junho de 2021. ISSN: 2448-0959, Link de acesso: https://www.nucleodoconhecimento.com.br/saude/doenca-de-hashimoto, DOI: 10.32749/nucleodoconhecimento.com.br/saude/doenca-de-hashimoto

\section{RESUMO}

Contexto: A Tireoidite de Hashimoto, principal causa de hipotireoidismo, é uma doença autoimune, caracterizada pelo caráter inflamatório da tireoide, que se manifesta por diversos sintomas sistêmicos, que variam de quadros leves a graves, como a encefalopatia de Hashimoto. Objetivo: Avaliar as repercussões fisiopatológicas e clínicas da Tireoidite de Hashimoto, com base nos dados da literatura vigente. Métodos: $O$ estudo configura uma revisão bibliográfica integrativa, fundamentada segundo a análise de 10 artigos, nacionais e internacionais, disponíveis na plataforma Biblioteca Virtual de Saúde. Resultados: Identificou-se que suscetibilidade genética e presença de níveis séricos reduzidos de vitamina $D$, zinco, ferro e selênio podem ser correlacionados com a probabilidade de acometimento pela Tireoidite de Hashimoto. No que tange aos exames de imagem, observou-se que o aumento progressivo do bócio está frequentemente relacionado a níveis séricos de FT3 (triiodotironina livre) altos, FT4 (tiroxina livre) baixos e alta proporção de FT3/FT4, o que despontou a hipótese de benefício da utilização de Levotiroxina nestes pacientes. No contexto imunológico foi observado que a presença de neutrofilia, plaquetose e níveis elevados de cortisol e estrogênio podem correlacionar-se à autoimunidade. Conclusão: Diversas condições influenciam no acometimento pela Tireoidite de Hashimoto e, sendo assim, faz-se necessário compreender a fisiopatologia e a clínica da doença para melhor intervir no manejo clínico.

Palavras-chave: Doença de Hashimoto, Sinais Clínicos, Fisiologia.

RC: 88463

Disponível em: https://www.nucleodoconhecimento.com.br/saude/doenca-de-hashimoto 


\section{INTRODUÇÃO}

A tireoidite de Hashimoto é atualmente a enfermidade autoimune mais prevalente, a doença endócrina de maior frequência e a principal causa de hipotireoidismo nas populações com acesso ao iodo em sua alimentação (WOJTAS; WADOLOWSKA; BANDURSKA-STANKIWWICZ, 2019) (CATUREGLI; DE REMIGIS; ROSE, 2014). Tende a afetar cerca de dez vezes mais a população feminina do que a masculina (TAYLOR et al., 2018) e sua etiologia pode ser classificada em primária ou secundária (CATUREGLI; DE REMIGIS; ROSE, 2014).

A forma primária compreende: manifestação clássica, vinculada à presença de anticorpos antitireodianos; variante fibrótica; variante relacionada à IgG4; hashitoxicose; forma juvenil; e a tireoidite silenciosa. Essas variações possuem em comum um aumento no tamanho da tireoide e a presença de um infiltrado linfocitário em seu tecido (CATUREGLI; DE REMIGIS; ROSE, 2014). As formas secundárias são iatrogênicas, relacionados a drogas imunomodulatórias, que eliciam a atividade do sistema imune, como o uso de interferon gama para o tratamento de hepatite $\mathrm{C} \mathrm{e}$ drogas modernas para o tratamento de neoplasias (CATUREGLI; DE REMIGIS; ROSE, 2014) (TAYLOR et al., 2018).

As manifestações clínicas da tireoidite de Hashimoto constituem o quadro de hipotireoidismo, cujo diagnóstico é laboratorial, com valores de TSH acima dos valores de referência concomitantes a valores de T4 (tiroxina) abaixo da normalidade (TAYLOR et al., 2018) (CHAKER et al., 2017). Fadiga, intolerância ao frio e rouquidão exemplificam aspectos do quadro clínico decorrente dessa alteração hormonal, que envolvem múltiplos sistemas. No trato grastrointestinal, há redução do peristaltismo e consequente constipação. Além disso, acúmulo de ácido hialurônico e glicosaminoglicanos torna a pele seca, endurecida e amarelada (CATUREGLI; DE REMIGIS; ROSE, 2014) (TAYLOR et al., 2018). Pode-se citar bradicardia, menor contratilidade do tecido muscular cardíaco e aumento da resistência vascular periférica constituem impactos no sistema cardiovascular (CHAKER et al., 2017).

$\mathrm{RC}: 88463$

Disponível em: https://www.nucleodoconhecimento.com.br/saude/doenca-de-hashimoto 
Anemia é também componente da clínica do hipotireoidismo, por influência no sistema hematopoético. No sexo feminino, pode ocorrer menorragia ou amenorreia, enquanto no sexo masculino a enfermidade está relacionada a teratozooespermia e redução da mobilidade dos espermatozoides (CATUREGLI; DE REMIGIS; ROSE, 2014) (LA VIGNERA; VITA, 2018). Em termos psiquiátricos e neurológicos, o hipotireoidismo pode envolver perda de memória e depressão, além de estar relacionado a uma condição rara, a encefalopatia de Hashimoto.

A relevância epidemiológica dessa patologia e a complexidade de suas manifestações sistêmicas justificam a elaboração do presente artigo com o escopo de revisar as repercussões fisiopatológicas e clínicas da doença de Hashimoto, a fim de elucidar dados da literatura sobre a apresentação da enfermidade e avanços na sua compreensão, proporcionando melhor prognóstico aos pacientes a partir do reconhecimento de suas necessidades de saúde.

Segundo Chaker et al. (2017), por exemplo, a literatura é consolidada em termos dos impactos cardiovasculares do hipotireoidismo, o que motiva o presente artigo a arrolar informações acerca de avanços na compreensão de outros aspectos da enfermidade, como a etiopatogenia e relevância clínica da menorragia, a redução no risco de hipotireoidismo associado ao fumo de tabaco, entre outros, além de revisitar aspectos consolidados da enfermidade.

\section{METODOLOGIA}

Trata-se de uma revisão bibliográfica do tipo bibliográfica integrativa de caráter retrospectivo com abordagem qualitativa, com intuito descritivo de estudos nacionais e internacionais. Além disso, a natureza desta pesquisa enquadra-se como aplicada, tendo em vista que busca compreender as repercussões fisiopatológicas e clínicas da doença de Hashimoto.

A pesquisa foi feita utilizando as bases de dados online Portal da Biblioteca Virtual em Saúde (BVS) e seus sites aliados. Inicialmente, foi realizada uma confirmação dos RC: 88463

Disponível em: https://www.nucleodoconhecimento.com.br/saude/doenca-de-hashimoto 
descritores pertinentes para abordar esta temática no corpo de trabalho por meio do vocabulário disponível no DeCS (Descritores em Ciências da Saúde), sendo esses: "doença de hashimoto", "fisiologia" e "sinais clínicos" nos idiomas português, inglês e espanhol. Além disso, em busca de aperfeiçoar a fórmula de busca, utilizou-se os sinônimos pertinentes e operadores booleanos, sendo tais: parênteses, AND e OR.

Por conseguinte, foram filtrados, em ordem sequencial citada, os artigos que estavam disponíveis na íntegra, indexados com recorte temporal contido nos últimos cinco anos e nos idiomas espanhol, inglês e português. De acordo com os resultados obtidos a partir da fórmula de busca, foram encontrados 40 artigos na BVS.

Os critérios de inclusão empregados foram: artigos que elucidassem pacientes, crianças ou adultos, que apresente doença de hashimoto e que aborde as principais repercussões fisiopatológicas e clínicas desta patologia, bem como as complicações para tal doença, inclusas no recorte temporal pré-determinado. Ressalta-se que a inclusão desses pacientes independia de etnia ou sexo. Excluíram-se artigos que abordassem somente pacientes com doença de Hashimoto isolada, sem repercussões. Além de artigos de opinião, estudos duplicados e artigos que não estavam disponíveis na íntegra, metanálises e revisões sistemáticas, estudos observacionais e de incidência e relatos de casos.

Diante disso, os pesquisadores, ISO e RMR, avaliaram todos os títulos e resumos dos artigos e selecionaram os estudos baseados nos critérios de elegibilidade anteriormente citados. Com isso, restou-se 10 artigos na BVS que contemplavam o objetivo da respectiva pesquisa.

Cabe ressaltar que a pesquisa não apresenta caráter prático, não havendo, portanto, a necessidade de submissão ao Comitê de Ética em Pesquisa (CEP). Uma vez que não ocorreu manipulação de seres humanos de forma direta ou indiretamente.

RC: 88463

Disponível em: https://www.nucleodoconhecimento.com.br/saude/doenca-de-hashimoto 
Para a apresentação dos resultados desta revisão, os achados foram colocados em um quadro contendo os principais aspectos técnicos dos artigos selecionados, sendo esses: título, ano e local de publicação, autoria e principais resultados.

\section{RESULTADOS}

Quadro 1: Principais Resultados dos Artigos Selecionados Para Pesquisa

\begin{tabular}{|c|c|c|c|}
\hline & Título & $\begin{array}{l}\text { Autores, } \\
\text { ano de } \\
\text { publicação } \\
\text { e país }\end{array}$ & Principais resultados \\
\hline ARTIGO & $\begin{array}{lr}\text { Oxidative } & \text { stress } \\
\text { markers, } & \text { trace } \\
\text { elements, and } \\
\text { endocrine } \\
\text { disrupting } \\
\text { chemicals } \\
\text { children in } \\
\text { Hashimoto's } \\
\text { thyroiditis. }\end{array}$ & $\begin{array}{l}\text { Sur et al., } \\
2019 \text {, } \\
\text { Turquia. }\end{array}$ & $\begin{array}{l}\text { Não foi comprovada relação entre o } \\
\text { estresse oxidativo e a Tireoidite de } \\
\text { Hashimoto. No entanto, a carência } \\
\text { de iodo e de zinco, a suscetibilidade } \\
\text { genética e a predominância do } \\
\text { desequilíbrio antioxidante/oxidante } \\
\text { em crianças foram considerados } \\
\text { fatores relevantes na constituição da } \\
\text { síndrome. }\end{array}$ \\
\hline $\begin{array}{l}\text { ARTIGO } \\
\|\end{array}$ & $\begin{array}{l}\text { The association } \\
\text { between thyroid } \\
\text { hormone balance } \\
\text { and thyroid volume } \\
\text { in patients with } \\
\text { Hashimoto } \\
\text { thyroiditis. }\end{array}$ & $\begin{array}{l}\text { Kawasaki et } \\
\text { al., 2019, } \\
\text { Japão. }\end{array}$ & $\begin{array}{l}\text { A relação de proporção entre } \\
\text { FT3/FT4 séricos se eleva conforme } \\
\text { o tamanho aumentado do bócio, não } \\
\text { tendo relação com idade, sexo e } \\
\text { TRAb, o que demonstra que o } \\
\text { tratamento com levotiroxina é capaz } \\
\text { de reduzir esta proporção. }\end{array}$ \\
\hline $\begin{array}{l}\text { ARTIGO } \\
\text { III }\end{array}$ & $\begin{array}{l}\text { The association } \\
\text { between thyroid } \\
\text { echogenicity and }\end{array}$ & $\begin{array}{l}\text { Jeong et al., } \\
2019 \text {, }\end{array}$ & $\begin{array}{l}\text { Quanto maior a reflexão das ondas } \\
\text { de ultrassom, mais frequentemente }\end{array}$ \\
\hline
\end{tabular}

RC: 88463

Disponível em: https://www.nucleodoconhecimento.com.br/saude/doenca-de-hashimoto 


\begin{tabular}{|c|c|c|c|}
\hline & $\begin{array}{l}\text { thyroid function in } \\
\text { pediatric and } \\
\text { adolescent } \\
\text { Hashimoto's } \\
\text { thyroiditis. }\end{array}$ & $\begin{array}{l}\text { Coreia do } \\
\text { Sul. }\end{array}$ & $\begin{array}{l}\text { foi observada a diminuição da } \\
\text { função tireoidiana. }\end{array}$ \\
\hline $\begin{array}{l}\text { ARTIGO } \\
\text { IV }\end{array}$ & $\begin{array}{l}\text { Multiple nutritional } \\
\text { factors and thyroid } \\
\text { disease, with } \\
\text { particular reference } \\
\text { to autoimmune } \\
\text { thyroid disease. }\end{array}$ & $\begin{array}{l}\text { Rayman, } \\
2018, \\
\text { Inglaterra. }\end{array}$ & $\begin{array}{l}\text { Doenças autoimunes da tireoide } \\
\text { estão diretamente ligadas a } \\
\text { comorbidades que geram } \\
\text { deficiências nutricionais de ferro, } \\
\text { selênio e iodo. Assim, a carência } \\
\text { destes nutrientes implica em } \\
\text { prejuízo da função tireoidiana. }\end{array}$ \\
\hline ARTIGO & $\begin{array}{lr}\text { Neutrophil- } & \text { and } \\
\text { Platelet- } & \text { to } \\
\text { Lymphocyte } & \text { Ratio } \\
\text { in Patients with } \\
\text { Euthyroid } \\
\text { Hashimoto's } \\
\text { Thyroiditis. }\end{array}$ & $\begin{array}{l}\text { Bilge et al., } \\
2019, \\
\text { Turquia. }\end{array}$ & $\begin{array}{l}\text { Foi observada neutrofilia e } \\
\text { plaquetose em pacientes com } \\
\text { Tireoidite de Hashimoto. }\end{array}$ \\
\hline $\begin{array}{l}\text { ARTIGO } \\
\text { VI }\end{array}$ & $\begin{array}{l}\text { Multiple nutritional } \\
\text { factors and the risk } \\
\text { of Hashimoto's } \\
\text { Thyroiditis. }\end{array}$ & $\begin{array}{l}\text { Shiqian e } \\
\text { Rayman, } \\
\text { 2017, Reino } \\
\text { Unido }\end{array}$ & $\begin{array}{l}\text { Fatores nutricionais como vitamina } \\
\text { D, selênio e ferro influenciam } \\
\text { diretamente na Tireoidite de } \\
\text { Hashimoto e, portanto, identificou-se } \\
\text { que a reposição destes nutrientes } \\
\text { pode ser de grande valia. }\end{array}$ \\
\hline $\begin{array}{l}\text { ARTIGO } \\
\text { VII }\end{array}$ & $\begin{array}{l}\text { The association of } \\
\text { elevated plasma } \\
\text { cortisol and } \\
\text { Hashimoto's } \\
\text { Thyroiditis, a }\end{array}$ & $\begin{array}{l}\text { Agha- } \\
\text { Hosseini et } \\
\text { al., 2016, } \\
\text { Irã. }\end{array}$ & $\begin{array}{l}\text { Notou-se que o cortisol estaria } \\
\text { elevado na Tireoidite de Hashimoto } \\
\text { e que glicocorticoides agem como } \\
\text { imunossupressores. }\end{array}$ \\
\hline
\end{tabular}

RC: 88463

Disponível em: https://www.nucleodoconhecimento.com.br/saude/doenca-de-hashimoto 


\begin{tabular}{|c|c|c|c|}
\hline & $\begin{array}{l}\text { neglected part of } \\
\text { immune response. }\end{array}$ & & \\
\hline ARTIGO & $\begin{array}{l}\text { Risk profile } \\
\text { analysis and } \\
\text { complications after } \\
\text { surgery for } \\
\text { autoimmune } \\
\text { thyroid disease. }\end{array}$ & $\begin{array}{l}\text { Thomusch } \\
\text { et al., 2018, } \\
\text { Alemanha. }\end{array}$ & $\begin{array}{l}\text { Observou-se que é uma cirurgia } \\
\text { segura com taxas de complicações } \\
\text { gerais } \quad \text { (tromboembólicas, } \\
\text { pulmonares, cardíacas) e } \\
\text { específicas (paralisia de cordas } \\
\text { vocais, hipoparatireoidismo } \\
\text { permanente) muito baixas. }\end{array}$ \\
\hline $\begin{array}{l}\text { ARTIGO } \\
\text { IX }\end{array}$ & $\begin{array}{l}\text { Hashimoto's } \\
\text { thyroiditis predicts } \\
\text { outcome in } \\
\text { intrathyroidal } \\
\text { papillary thyroid } \\
\text { cancer. }\end{array}$ & $\begin{array}{l}\text { Marotta et } \\
\text { al., 2017, } \\
\text { Itália. }\end{array}$ & $\begin{array}{l}\text { Foi observado que o câncer papilar } \\
\text { de tireoide se dá de maneira } \\
\text { independente da tireoidite de } \\
\text { Hashimoto e, por isso, não é capaz } \\
\text { de viabilizar um prognóstico } \\
\text { assertivo. }\end{array}$ \\
\hline $\begin{array}{l}\text { ARTIGO } \\
X\end{array}$ & $\begin{array}{l}\text { Evidence of } \\
\text { impaired } \\
\text { carbohydrate } \\
\text { assimilation in } \\
\text { euthyroid patients } \\
\text { with Hashimoto's } \\
\text { thyroiditis. }\end{array}$ & $\begin{array}{l}\text { Heckl et al., } \\
2015, \\
\text { Londres. }\end{array}$ & $\begin{array}{l}\text { Este estudo evidenciou que a má } \\
\text { absorção de carboidratos e as } \\
\text { complicações gastrointestinais são } \\
\text { frequentes em pacientes } \\
\text { eutireoideos com tireoidite de } \\
\text { Hashimoto. }\end{array}$ \\
\hline
\end{tabular}

Fonte: Autoria Própria, 2021.

Observou-se, dentre os estudos analisados, que a associação entre o estresse oxidativo e Tireoidite de Hashimoto (TH) ainda é controversa. Dentre os artigos selecionados, 30\% evidenciaram uma correlação entre a deficiência de iodo e a Tireoidite de Hashimoto, haja visto a detecção de deficiência deste mineral em pacientes portadores da doença autoimune. Ademais, fatores como a suscetibilidade

$\mathrm{RC}: 88463$

Disponível em: https://www.nucleodoconhecimento.com.br/saude/doenca-de-hashimoto 
genética e a presença de níveis séricos reduzidos de zinco e vitamina $D$ também foram identificados entre os indivíduos acometidos (SUR et al., 2019).

Em contrapartida, notou-se que a ingestão abundante de iodo também aumenta a probabilidade de indução de uma tireoidite autoimune. Neste contexto, estudo de $\mathrm{Hu}$ et al. (2017) alegou que a ingesta adequada de selênio seria a conduta ideal a ser adotada por indivíduos que habitam áreas com dieta sabidamente deficiente ou abundante em iodo. Ainda em relação às carências nutricionais, identificou-se que algumas comorbidades, tais como bócio nodular, doença celíaca, gastrite autoimune e hipotireoidismo podem ser desencadeadas pelas deficiências nutricionais de ferro, iodo e selênio, nutrientes que também desempenham papel significativo na manutenção da função tireoidiana e que, portanto, são necessários à homeostase endocrinológica (RAYAMAN et al., 2018).

Identificou-se, ainda, uma correlação entre a moléstia autoimune e o acometimento gastrointestinal, mediante a observação de que pacientes portadores de Tireoidite de Hashimoto eutireoidiana sofrem, constantemente, de má absorção de carboidratos e, por conseguinte, apresentam sintomas gastrointestinais, como constipação, meteorismo, lentificação do esvaziamento gástrico, motilidade reduzida das vísceras, diminuição das frequências de fezes ou desenvolvimento de íleo paralítico (em casos mais severos). Concluiu-se, portanto, que a má assimilação de carboidratos configura um novo fator de risco para a autoimunidade em geral e, em particular, para a Tireoidite de Hashimoto (HECKL et al., 2015).

Dentre os estudos avaliados, foi possível delinear a existência de sinais característicos da Tireoidite de Hashimoto, exemplificados pelo hipotireoidismo e pela diminuição da ecogenicidade da glândula tireóide quando avaliada através de ultrassonografia cervical. No tocante ao exame ultrassonográfico, notou-se correlação entre a redução da ecogenicidade da glândula e o nível mais elevado de TSH em pacientes pediátricos e adolescentes, o que infere insuficiência tireoidiana (JEONG et al., 2019). 
No que se refere aos exames de imagem, aferiu-se que pacientes com tomografia computadorizada evidenciando aumento progressivo do bócio apresentaram, mais frequentemente, níveis séricos de FT3 (triiodotironina livre) altos, FT4 (tiroxina livre) baixos e uma alta proporção FT3/FT4, causada pela elevação da atividade de desiodase tireoidiana (preferencialmente a atividade de desiodase tireoidiana tipo 2). As relações séricas FT3/FT4 constituem uma associação positiva com o volume da tireoide, independentemente da idade, do sexo, do índice de massa corporal e dos níveis de TgAb (anticorpo anti-tireoglobulina) e de TSH (hormônio estimulador da tireóide). Nesta perspectiva, foram realizados ensaios com o uso de Levotiroxina, com o fito de observar o efeito da redução do volume tireoidiano nestes pacientes (KAWASAKI et al., 2019).

No que concerne ao mecanismo de autoimunidade, foi elucidada a existência de neutrofilia e plaquetose em pacientes portadores da Tireoidite de Hashimoto. Verificou-se, portanto, que a proporção de neutrófilos para linfócitos (NLR) e a proporção de plaquetas para linfócitos (PLR) são mais elevadas em pacientes com Tireoidite de Hashimoto eutireoidiano sem qualquer terapia de reposição e constituem, possivelmente, indicadores não específicos de distúrbios imunológicos. Tal associação foi considerada positiva apenas entre NLR e PLR, sendo explicada pela queda na contagem de linfócitos durante a inflamação (BILGE et al., 2019).

Por sua vez, foram mensurados níveis elevados de cortisol em pacientes portadores da Tireoidite Autoimune. Tal hormônio tem função imunomoduladora e imunossupressora e, portanto, é capaz de regular negativamente o sistema imunológico. Logo, quando associados à idade, estes níveis elevados de glicocorticóides podem favorecer o desenvolvimento da autoimunidade da glândula tireoidiana. Ainda neste cenário, o estrogênio também demonstrou apresentar papel fundamental na modulação imunológica e, por isso, constitui uma causa da maior frequência de doenças autoimunes em mulheres (HOSSEINI et al., 2016).

RC: 88463

Disponível em: https://www.nucleodoconhecimento.com.br/saude/doenca-de-hashimoto 
Em consonância com os estudos, contemplou-se que a segurança da cirurgia para doenças autoimunes da tireóide é maior quando comparada à cirurgia para doenças multinodulares. Os estudos apontaram que após a cirurgia para doença autoimune da tireoide a taxa de complicações pós-operatórias gerais, tais como infecção de ferida, acometimentos cardíacos e eventos tromboembólicos não aumentou e, de maneira oposta, foi tão baixa quanto a cirurgia para bócio multinodular benigno. Entretanto, percebeu-se um risco aumentado para o desenvolvimento de hipoparatireoidismo após a cirurgia de abordagem da TH (THOMUSCH et al., 2021).

Por fim, buscou-se demonstrar algum grau de correlação entre progressão de câncer papilífero de tireoide e a presença de Tireoidite Autoimune. No entanto, os achados não evidenciaram conexão entre o comportamento do tumor e a comorbidade autoimune e, portanto, fora comprovado que a existência de $\mathrm{TH}$ em pacientes portadores da neoplasia não é capaz de melhorar a especificidade prognóstica (MAROTTA et al., 2017).

\section{DISCUSSÃO}

\section{COMPLICAÇÕES DA TIREOIDITE DE HASHIMOTO}

Pacientes com a Doença de Hashimoto podem eventualmente evoluir com sintomas de hipotireoidismo. Isso acontece quando a resposta autoimune é suficiente para causar a destruição celular da tireóide, permitindo que esses sintomas sejam insidiosos, variáveis e,

muitas vezes, sistêmicos (AHMED; AL-SHAIKH; AKHTAR, 2020).

Apesar de o mixedema ser a característica clássica da pele associada ao hipotireoidismo, esse sinal é incomum e ocorre apenas em casos graves. Nessas situações, a pele pode se tornar escamosa e seca, especialmente nas superfícies extensoras, palmas das mãos e plantas dos pés. Além disso, no exame histológico, pode-se verificar o afinamento da epiderme (AHMED; AL-SHAIKH; AKHTAR, 2020).

$\mathrm{RC}: 88463$

Disponível em: https://www.nucleodoconhecimento.com.br/saude/doenca-de-hashimoto 
Com a progressão da doença, há uma redução da taxa de crescimento do cabelo e tal fânero pode tornar-se seco, áspero, opaco e quebradiço. Portanto, alopecia parcial ou até mesmo difusa não é incomum em pacientes com Tireoidite de Hashimoto (AHMED; AL-SHAIKH; AKHTAR, 2020).

A diminuição da função tireoidiana também é capaz de aumentar a resistência vascular periférica em até $50 \%$ a $60 \%$ e reduzir o débito cardíaco em até $30 \%$ a $50 \%$. Dessa forma, a bradicardia pode ser resultante da perda da ação cronotrópica do hormônio tireoidiano diretamente nas células sinotariais. De acordo com Akamizu e Amino (2017), a doença em questão predispõe à doença vascular, assim como à oclusão coronariana, uma vez que se acredita que os elevados títulos de autoanticorpos tireoidianos e as alterações morfológicas da tireoidite acometem mais frequentemente os pacientes com doença arterial coronariana. Contudo, como foi citado acima, a maioria dos pacientes apresenta algum sintoma diretamente relacionado ao sistema cardiovascular (AHMED; AL-SHAIKH; AKHTAR, 2020; AKAMIZU; AMINO, 2017).

Pode-se citar também queixas musculoesqueléticas, incluindo dor torácica, fibrosite e artrite reumatóide, as quais acometem cerca de $25 \%$ dos pacientes. Todavia, ressaltase que qualquer um dos sintomas musculoesqueléticos comuns ao hipotireoidismo pode ocorrer da mesma forma (AKAMIZU; AMINO, 2017).

As complicações neurológicas da Doença de Hashimoto estão, muitas vezes, associadas à disfunção da glândula tireoide, porém a encefalopatia ou encefalite de Hashimoto geralmente é encontrada em pacientes eutireoidianos. Essa encefalopatia é uma complicação muito rara relacionada aos altos títulos de autoanticorpos tireoidianos, mas é tratável, responsiva a esteroides, progressiva ou recidivante. Ela pode se apresentar de forma subaguda ou aguda com convulsões, episódios similares a AVE, mioclonia e tremor. No que tange aos exames complementares, nessa condição, encontra-se um EEG anormal e proteínas do LCR sem pleocitose (AKAMIZU; AMINO, 2017).

$\mathrm{RC}: 88463$

Disponível em: https://www.nucleodoconhecimento.com.br/saude/doenca-de-hashimoto 
De acordo com uma pesquisa publicada no International Journal of Endocrinology (2014), a Tireoidite de Hashimoto pode interferir nos mecanismos de ovulação e no metabolismo dos hormônios sexuais. Consequentemente, ela pode afetar a fertilidade e, se houver gravidez, aumentar a incidência de perda gestacional precoce e afetar negativamente a saúde do feto.

Além disso, a Tireoidite de Hashimoto, e o eventual hipotireoidismo causado por essa patologia, podem ser comumente associados a outras várias condições, a exemplo da doença de Addison, diabetes mellitus, hipogonadismo, hipoparatireoidismo, anemia perniciosa, vitiligo, urticária e Síndrome de Sjogren. Por outro lado, algumas síndromes mais incomuns já foram descritas também, tais como: depósitos amilóides na tireoide e pneumonite intersticial linfocítica responsiva a esteróides (AKAMIZU; AMINO, 2017).

\section{CONCLUSÃO}

Dentre os estudos analisados nesta revisão, nenhum atestou a comprovação da relação entre o estresse oxidativo e a Tireoidite de Hashimoto. Contudo, pode-se observar com clareza que fatores como a predominância genética a carência de elementos, dentre eles, o iodo e o zinco, e a preponderância do desequilíbrio antioxidante/oxidante em crianças foram considerados causas relevantes na constituição da síndrome.

Além disso, ficou nítida que a relação de proporção entre os níveis de FT3/FT4 séricos presentes no sangue aumentou conforme o tamanho elevado do bócio e não sofreu alteração relacionada a sexo, TRAb e idade.

Outrossim, os estudos mostraram que as doenças autoimunes da tireoide estão de modo direto correlacionadas às comorbidades acarretadas por deficiências nutricionais de ferro, selênio e iodo. Dessa forma, a carência destes nutrientes demonstrou causar prejuízo na função tireoidiana, o que infere que a reposição destes fatores é de relevância terapêutica.

RC: 88463

Disponível em: https://www.nucleodoconhecimento.com.br/saude/doenca-de-hashimoto 
Em outra análise, no que concerne ao mecanismo de autoimunidade, foi exposta a existência de neutrofilia e plaquetose em pacientes portadores da Tireoidite de Hashimoto. Foi ratificada também, portanto, a relação de proporção entre neutrófilos e linfócitos (NLR) como também a fração de plaquetas para linfócitos (PLR). Demonstrou-se, ainda, a vinculação da má assimilação de carboidratos como um novo elemento de risco para a autoimunidade em diversas condições e, em especial, para a Tireoidite de Hashimoto.

Identifica-se, portanto, que a discussão acerca das repercussões fisiopatológicas inerentes à Tireoidite de Hashimoto é fator crucial para o desenvolvimento de novas propostas de intervenção terapêutica e manejo diagnóstico da síndrome.

\section{REFERÊNCIAS}

AGHA-HOSSEINI, Farzaneh et al. The association of elevated plasma cortisol and Hashimoto's Thyroiditis, a neglected part of immune response. Acta Clin Belg, Irã, 6 fev. 2016. Disponível em: https://pesquisa.bvsalud.org/portal/resource/pt/mdl27075805. Acesso em: 1 abr. 2021.

BILGE, Muge et al. Neutrophil- and Platelet- to Lymphocyte Ratio in Patients with Euthyroid Hashimoto's Thyroiditis. Exp Clin Endocrinol Diabetes, Turquia, 1 set. 2019. Disponível em: https://pesquisa.bvsalud.org/portal/resource/pt/mdl-30267388. Acesso em: 1 abr. 2021.

CATUREGLI, P.; DE REMIGIS, A.; ROSE, N. R. (2014). Hashimoto thyroiditis: Clinical and diagnostic criteria. Autoimmunity Reviews, 13(4-5), 391-397. doi:10.1016/j.autrev.2014.01.00. Acesso em: 1 abr. 2021.

CHAKER, L.; BIANCO, A. C.; JONKLAAS, J.; PEETERS, R. P. (2017). Hypothyroidism. The Lancet, 390(10101), 1550-1562. doi:10.1016/s01406736(17)30703-1. Acesso em: 1 abr. 2021.

$\mathrm{RC}: 88463$

Disponível em: https://www.nucleodoconhecimento.com.br/saude/doenca-de-hashimoto 
HECKL, S et al. Evidence of impaired carbohydrate assimilation in euthyroid patients with Hashimoto's thyroiditis. European Journal of Clinical Nutrition, Londres, 7 out. 2015. Disponível em: https://www.nature.com/articles/ejcn2015167.pdf. Acesso em: 1 abr. 2021.

JEONG, Sun. The association between thyroid echogenicity and thyroid function in pediatric and adolescent Hashimoto's thyroiditis. Medicine (Baltimore), Coreia do Sul, 1 abr. 2019. Disponível em: https://pesquisa.bvsalud.org/portal/resource/pt/mdl30946351. Acesso em: 1 abr. 2021.

KAWASAKI, Motoki et al. The association between thyroid hormone balance and thyroid volume in patients with Hashimoto thyroiditis. Endocr J, Japão, 1 ago. 2019. Disponível em: https://pesquisa.bvsalud.org/portal/resource/pt/mdl-31155540. Acesso em: 1 abr. 2021.

MAROTTA, Vincenzo et al. Hashimoto 's thyroiditis predicts outcome in intrathyroidal papillary thyroid cancer. Endocr Relat Cancer, Itália, 10 set. 2017. Disponível em: https://pesquisa.bvsalud.org/portal/resource/pt/mdl-28696209. Acesso em: 1 abr. 2021.

RAYMAN, Margaret et al. Multiple nutritional factors and thyroid disease, with particular reference to autoimmune thyroid disease. Proc Nutr Soc, Inglaterra, 13 set. 2018. Disponível em: https://pesquisa.bvsalud.org/portal/resource/pt/mdl-30208979. Acesso em: 1 abr. 2021

SHIQIAN, Hu; RAYMAN, M.P. Multiple nutritional factors and the risk of Hashimoto's Thyroiditis. Exp Clin Endocrinol Diabetes, Reino Unido, 1 mai. 2017. Disponível em: https://pesquisa.bvsalud.org/portal/resource/pt/mdl-28290237. Acesso em: 1 abr. 2021.

SUR7, Unzile et al. Oxidative stress markers, trace elements, and endocrine disrupting chemicals in children with Hashimoto 's thyroiditis. Toxicol Mech Methods, Turquia,

$\mathrm{RC}: 88463$

Disponível em: https://www.nucleodoconhecimento.com.br/saude/doenca-de-hashimoto 
29 jul. 2019. Disponível em: https://pesquisa.bvsalud.org/portal/resource/pt/mdl31354016. Acesso em: 1 abr. 2021.

TAYLOR, P. N. et al. Global epidemiology of hyperthyroidism and hypothyroidism. Nature Reviews Endocrinology, 14(5), 301-316. doi:10.1038/nrendo.2018.18. Acesso em: 1 abr. 2021.

THOMUSCH, $\mathrm{O}$ et al. Risk profile analysis and complications after surgery for autoimmune thyroid disease. Br J Surg, Alemanha, 6 maio 2018. Disponível em: https://pesquisa.bvsalud.org/portal/resource/pt/mdl-29579336. Acesso em: $1 \mathrm{abr}$. 2021.

WOJTAS, N.; WADOLOWSKA, L.; BANDURSKA-STANKIEWLCZ, E. Evaluation of Qualitative Dietary Protocol (Diet4Hashi) Application in Dietary Counseling in Hashimoto Thyroiditis: Study Protocol of a Randomized Controlled Trial. Int. J. Environ. Res. Public Health 2019, 16, 4841. https://doi.org/10.3390/ijerph16234841. Acesso em: 1 abr. 2021.

Enviado: Maio, 2021.

Aprovado: Junho, 2021. 